\title{
Medicamentos Quirais: da dimensão química à discussão política
}

\author{
Chiral Drugs: from the chemical dimension \\ to political discussion
}

Jorge A. Z. Bermudez 1

Paulo Barragat 2

1 Departamento de Ciências Biológicas, Escola Nacional de Saúde Pública, Fiocruz. Rua Leopoldo Bulhões 1480, Rio deJaneiro, RJ 21041-210, Brasil.

2 Associação Brasileira das Indústrias de Química Fina. Rua Nilo Peçanha 151,

Rio de Janeiro, RJ

20020-100, Brasil.
Resumo São discutidos aspectos conceituais dos medicamentos quirais, correspondendo a substâncias enanti omeri camente puras, constantes de apenas um dos i sômeros, dextrógi ro (R) ou levógiro (S). O impacto no mercado mundial, que hoje atinge um contingente de US\$ 35,6 bilhões, correspondente a aproxi madamente $20 \%$ do total, às custas de medi camentos qui rais, é ressaltado pel os autores como si gnificativo das tendências atuais. A expansão crescente do mesmo leva a crer que existe uma tendência níti da a quea produção de medicamentos quirais possa, em breve, representar uma nova forma de domíni o por parte das empresas hegemônicas, impactando definitivamente o mercado dos produtos sob proteção patentária e inviabilizando a política de crescimento mundial de medicamentos genéricos. Finalizando, é levantada a relação entre os medicamentos qui rais eas misturas racêmi cas, chamando a atenção para a necessi dade de incorporar esta dimensão química no âmbito da discussão das políticas públicas, consi derando também a capaci dade instalada em centros de pesqui sa e universidades brasil ei ras.

Palavras-chave Medicamentos Quirais; Política de Medicamentos; Indústria Farmacêutica; Mercado de Medicamentos; Processos Estereoquímicos

Abstract Conceptual aspects regarding chiral drugs are discussed referring to enantiomerically pure substances, containing only one isomer, with optical orientation to the right (R) or left (S). Impact on world market, that means today over US\$35 billions, or near $20 \%$ of total market with chiral drugs, is discussed as significative of actual trends. Growing expansion of this matter makes us think that there exists a clear trend that will lead chiral drugs to represent a new form of market domination, mainly regarding patent protection and bypassing crescent generic drug market policies. Finally, the questi on regarding chiral drugs and racemic mixtures is arised, calling attention for the need to incorporate this chemical dimension within health policy discussion, also considering brazi lian capacity in research centers and universities.

Key words Chiral Drugs; Drug Policies; Pharmaceutical Industry; Drug Market; Stereochemical Processing 


\section{Introdução}

A partir da constatação de que diferenças isoméricas podem dar origem a diferenças na atividade biológica, o reconhecimento e a possibilidade de desenvolvimento de estereoisômeros isolados passou a ser uma abordagem de importância para a indústria farmacêutica (Federsel, 1993). Durante a última década, a tendência para o desenvolvimento de substâncias estereoquimicamente puras passou a priorizar a identificação das diferenças na atividade biológica. Entretanto, desde 1874, quando a estrutura do átomo tetrahédrico do carbono foi proposta por Van't Hoff e se especulou sobre a relação entre atividade ótica e a constituição química, a síntese assimétrica constitui uma das áreas mais relevantes da Estereoquímica (Eliel, 1974).

Conforme Ferreira et al. (1996), as drogas racêmicas são misturas de enantiômeros, ou seja, contêm duas substâncias diferentes, que apresentam atividades biológicas também diferentes, podendo apresentar efeitos colaterais significativos. Principalmente quando obtidas a partir de síntese química, as substâncias são compostas por dois isômeros a partir do denominado centro quiral, sendo um dextrógiro e o outro levógiro. Considerando os isômeros como a imagem espelhada, cada um dos mesmos pode apresentar atividade biológica diferente. A quiral idade se coloca em contraposição aos produtos racêmicos, que normalmente apresentam quantidades equimolares de ambos enantiômeros. Em trabal ho anterior, chamamos a atenção para a importância de incorporar esta dimensão química à discussão política a respeito das tendências mercadológicas da indústria farmacêutica no mundo (Bermudez, 1995).

Tabela 1

Comparação entre a atividade biológica e a configuração absoluta de substâncias relacionadas enantiomericamente.

\begin{tabular}{|c|c|c|}
\hline Nome da substância & $\begin{array}{l}\text { Configuração dextrógira } \\
\text { (ou como especificado) }\end{array}$ & $\begin{array}{l}\text { Configuração levógira } \\
\text { (ou como especificado) }\end{array}$ \\
\hline Fenilalanina & Doce & Amargo \\
\hline Talidomida & Sedativo e hipnótico & $\begin{array}{l}\text { Extremamente } \\
\text { teratogênico }\end{array}$ \\
\hline Verapamil & Ação anti-tumoral & Ação antiarrítmica \\
\hline Cloranfenicol & $(\mathrm{R}, \mathrm{R})$ Antibacteriano & $(\mathrm{S}, \mathrm{S})$ Inativo \\
\hline Propranolol & Contraceptivo & $\begin{array}{l}\text { Anti-hipertensivo } \\
\text { antiarrítmico }\end{array}$ \\
\hline Etambutol & $(R, R)$ Causa cegueira & $(\mathrm{S}, \mathrm{S})$ Tuberculostático \\
\hline Deltametrina & $(\mathrm{R}, \mathrm{R}, \mathrm{S})$ Inseticida potente & $(\mathrm{S}, \mathrm{S}, \mathrm{R})$ Inativo \\
\hline 1-Cloropropano-2,3-diol & Tóxico & Atividadade anti-fértil \\
\hline Paclobutrazol & $(\mathrm{R}, \mathrm{R})$ Fungicida & $\begin{array}{l}(\mathrm{S}, \mathrm{S}) \text { Regulador do } \\
\text { crescimento de plantas }\end{array}$ \\
\hline
\end{tabular}

Fonte: Adaptado de Federsel (1993) e Chan (1993).
Utilizaremos, nesta publicação, a denominação de "medicamentos quirais" para identificar aqueles produtos enantiomericamente puros, constantes de apenas um dos isômeros, de orientação dextrógira (R) ou levógira (S).

Alguns produtos são bem conhecidos em Medicina como medicamentos quirais, embora não seja difundida a dimensão química da quiralidade na discussão da Saúde, nem se considera este conceito entre os marcos referenciais que norteiam a política de medicamentos. A seguir, apresentamos exemplos significativos de produtos conhecidos:

No que tange à possibilidade industrial de produzir moléculas definidas do ponto de vista estereoquímico, ou enantiômeros, podemos incluir (Federsel, 1993):

- Separação de misturas racêmicas por cristalização, cromatografia ou resolução cinética; - Utilização de compostos oticamente puros contendo estruturas presentes na natureza, isoladas de fontes naturais ou manufaturadas por processos enzimáticos ou similares;

- Utilização de produtos não-naturais, mas elaborados em laboratório, freqüentemente disponíveis com alta pureza enantiomérica; - Aplicação de síntese assimétrica, na qual os compostos pró-quirais são transformados, com a ajuda de microrganismos ou catalizadores, para moléculas de alta pureza ótica.

A estereoespecificidade é influenciada por fatores de importância para a otimização dos processos, tais como condições de reação, capacidade, equipamento, disponibilidade de matérias- primas e reagentes, segurança e condições ambientais e economia de escala. É necessário também proceder a uma série de técnicas analíticas para assegurar a qualidade dos produtos, principalmente quando há indicativos de que um determinado isômero é responsável por efeitos colaterais. A outrora utilizada determinação da rotação ótica pela polarimetria cedeu lugar a métodos altamente seletivos e sofisticados, como a cromatografia gasosa (GC), a cromatografia líquida de alto desempenho (HPLC), a espectroscopia por ressonância nuclear magnética (NMR) e as medidas cristalográficas utilizando raios- $X$.

Impacto no mercado mundial: tendências industriais

O desenvolvimento de medicamentos quirais passou a ter importância concreta a partir de novas diretrizes para registro adotadas a partir de 1992 pelo "Food and Drug Administration", órgão de controle dos E.U.A. (Stinson, 1992). 
Em decorrência, algumas empresas assumiram a decisão de desenvolver enantiômeros isolados. O autor considera que o desenvolvimento de medicamentos quirais pode vir a ter no futuro próximo um impacto semelhante ao desenvolvimento da Biotecnologia. Os avanços relativos à incorporação de medicamentos quirais no mercado são fundamentados na possibilidade de produzir quantidades industriais dos mesmos e nos problemas que se identificam nas misturas racêmicas, entre os quais se destacam as diferentes rotas bioquímicas de metabolização, diferenças individuais nas reações aos enantiômeros $\mathrm{R}$ e $\mathrm{S}$ e as reações adversas presentes em um dos mesmos. Deutsch (1991) lembra que, no futuro próximo, as misturas racêmicas, da mesma maneira que as atuais combinações de drogas, terão que ser justificadas.

Duas empresas que conseguiram integrar a produção de intermediários enantiomêricos com uma produção ampla na área da Química Fina são hoje responsáveis pelo fornecimento de quase a totalidade dos intermediários utilizados pela indústria farmacêutica mundial. São elas a Kamegafuchi Chemical Industry Co., no Japão, e a DSM Andeno, na Holanda e E.U.A. Outras empresas integram o mercado oferecendo serviços na área da Quirotecnologia. A tendência e a expansão crescente do mercado mundial sugerem que, nos próximos anos, esta indústria evolua para poucas dezenas de empresas, uniformemente capazes e com poucas diferenças entre si, a não ser nas dimensões (Stinson, 1994a).

Análise procedida por Polastro (Stinson, 1994b) estima que mudanças radicais irão se processar no mercado mundial de Química Fina nos próximos 15 anos. Destaca-se que a expansão do mercado dos medicamentos enantioméricos tende a crescer com rapidez devido ao maior valor agregado de sua produção. O crescimento do mercado mundial destes produtos é analisado como estreitamente relacionado com as reformas que muitos países implementam na atenção à saúde. Considerando as diversas alternativas que se planteiam, o autor conclui que a tendência da indústria farmo-química é evoluir para um sistema "racional", baseado em relações custo-benefício e não apenas em preços, implicando uma maior liberal ização por parte das agências governamentais, mas também acarretando uma profunda reestruturação do sistema de atenção à saúde, com a indústria se adaptando e competindo no âmbito do mesmo.

O redesenvolvimento de produtos, objetivando a transformação de misturas racêmicas em enantiômeros, apresenta-se como um campo em pleno desenvolvimento e com extraordinário potencial. Por um lado, há a questão do custo, estimando-se que, em um prazo de apenas quatro anos e com custo de US\$ 3 milhões, é possível transformar um produto racêmico, previamente licenciado, em uma nova substância enantiomericamente pura. Em contraposição, é estimado em 10 a 12 anos e investimentos da ordem de US\$240 milhões o custo do desenvolvimento de um novo produto. Por outro lado, existem muitos produtos racêmicos com proteção patentária expirada ou em vias de expirar, possibilitando às corporações transnacionais patentear enantiômeros de seus produtos racêmicos como estratégia para inviabilizar a concorrência promovida pelos produtos genéricos. Nesse sentido, estaria estabelecida uma nova reserva de mercado, duplicando os prazos legais de proteção patentária (Stinson, 1992; Ferreira et al., 1996). Efetivamente, enquanto determinadas empresas alegam que a pureza enantiomérica deve compatibilizar especificações técnicas com viabilidade econômica, outras procuram atingir elevadíssimos graus de pureza nos seus produtos quirais como estratégia para afastar permanentemente a competição dos produtores de medicamentos genéricos (Stinson, 1994a).

Este tipo de estratégia viria impedir a diminuição dos custos com a concorrência estabelecida no mercado de medicamentos genéricos, intercambiáveis e bioequivalentes, conforme recomendações da Organização Mundial da Saúde (WHO, 1993a, b). Há muita polêmica na contraposição entre produtos racêmicos e quirais, originada basicamente em função das características gerais do mercado farmacêutico mundial, às quais já nos referimos em trabalhos anteriores, chamando a atenção para o alto grau de oligopolização, concentração e internacionalização (Bermudez, 1992; 1994; 1995). Do ponto de vista mercadológico e de concorrência entre os produtos das grandes corporações transnacionais, Stinson (1992) analisa as vantagens que podem advir da transformação de produtos racêmicos em quirais, destacando benefícios clínicos, vantagens de mercado considerações vinculadas à esfera regulatória.

A primeira questão suscitada é se um determinado enantiômero é superior à mistura racêmica. É citado o exemplo do labetalol, beta-bloqueador licenciado nos Estados Unidos para o tratamento da hipertensão arterial. Em 1989, o produto quiral correspondente (R,R)-(-)-labetalol foi introduzido no Japão, com a indicação de antagonismo específico de receptores cardíacos beta 2, isento da afinidade por receptores alfa presente na mistura racêmica. Entretanto, hepatotoxicidade levou à sua retirada em menos de um ano. 
A segunda questão levantada é pertinente à real avaliação de vantagens na mudança para produtos quirais, citando o exemplo de determinadas categorias terapêuticas que se consolidaram no mercado, como é o caso dos antiinflamatórios não-esteróides. Neste caso, considerando os produtos e o número deles que se encontram competindo no mercado, uma mudança para um produto quiral, acarretando um custo mais alto, dificilmente seria absorvido sem demonstar efetivamente benefícios claros, como maior segurança. Complementarmente, encontram-se em vias de aprovação produtos que representam reais avanços terapêuticos, com os quais os novos medicamentos quirais teriam que competir.

O terceiro fator levantado refere-se ao fato de que dois enantiômeros podem apresentar ações farmacológicas distintas. O produto racêmico nebivolol, cujos enantiômeros apresentam respectivamente as ações de beta-bloqueador e vasodilatador, potencializam a ação anti-hipertensiva, com vantagens sobre a ação isolada de apenas um de seus isômeros.

Uma última questão se relaciona à controvérsia entre baixa dosagem e a capacidade do produto poder ser mais rapidamente inativado no organismo. Da mesma maneira, é necessário considerar determinadas formas farmacêuticas com al ta especificidade de ação, como é o caso de substâncias para liberação lenta e efeito terapêutico prolongado já desenvolvido como outra estratégia mercadológica que empresas líderes de mercado utilizam e que, não necessariamente, seriam substituídas por produtos quirais.

Outro aspecto que necessariamente deve ser levado em consideração, ao analisarmos o impacto que os medicamentos quirais possam vir a ter no futuro próximo, é a vertiginosa expansão no mercado mundial. Em 1992, era estimado que esses produtos já eram detentores de uma parcela de US\$ 18 bilhões, com um crescimento de $22 \%$ entre 1992 e 1993. Incluindo os produtos quirais já aprovados e os novos produtos a serem aprovados, é estimado queas vendas do mercado mundial de medicamentos quirais atinja cifras próximas aos US\$ 60 biIhões para o ano de 1997. Com base na manipulação dos processos metabólicos de microrganismos, antibióticos obtidos por processos fermentativos consistem de apenas um enantiômero (Stinson, 1992, 1993, 1994a).

A expansão desse mercado levou a que, em pouco mais de um ano, as vendas com produtos quirais dobrassem para um valor de US\$ 35 bilhões, conforme Tabela 2.
Tabela 2

Vendas mundiais de medicamentos quirais em 1993.

\begin{tabular}{lc}
\hline Classes Terapêuticas & Vendas (US\$ bilhões) \\
\hline Cardiovasculares & 11,3 \\
Antibióticos & 10,8 \\
Hormônios & 4,5 \\
Sistema Nervoso Central & 2,0 \\
Antiinflamatórios & 1,5 \\
Antitumorais & 1,0 \\
Outros & 4,5 \\
Total & 35,6 \\
\hline
\end{tabular}

Fonte: Stinson (1994a).

Perspectivas: da dimensão química à discussão política

Até recentemente, o desenvolvimento de misturas racêmicas era predominante entre as empresas produtoras com atuação na área de Pesquisa \& Desenvolvimento, por considerar que ambos enantiômeros eram igualmente ativos, ou um dos mesmos inativo e inócuo (Stinson, 1993). Diante dessa realidade, as etapas tecnológicas para a purificação de enantiômeros acrescia consideravelmente os custos, sem justificativa tecnológica, clínica ou mercadológica. Atualmente, a tendência a produzir alterações nas misturas racêmicas vem crescendo em importância. Um exemplo é a comercialização do analgésico ibuprofeno, sob várias marcas e por várias empresas como mistura racêmica. O desenvolvimento do (S)-(+)-enantiômero o transformou em produto a ser comercializado como medicamento de venda livre ("over-the-counter" ou OTC), com vantagens mercadológicas e com estimativas de atingir um mercado de um bilhão de dólares anuais (Stinson, 1994). A descoberta de efeitos farmacológicos opostos em enantiômeros de um mesmo produto racêmico se constitui hoje na maior motivação para proceder ao desenvolvimento do produto quiral.

Por um lado, então, constatamos que indústrias farmacêuticas estão implementando a transformação de produtos racêmicos em quirais, com a tentativa de transformá-los em medicamentos não sujeitos à prescrição médica (OTC) ou de venda livre, menos sujeitos ao rigor das formalidades regulatórias.

Por outro lado, mencionamos a possibilidade de que detentoras de patentes farmacêuticas podem ampliar os prazos legais de proteção de seus produtos ao promover o patenteamento do medicamento quiral, criando uma nova e mais duradoura reserva de mercado, 
com aumento de preço e combatendo a expansão do mercado de produtos genéricos.

A análise da questão dos medicamentos quirais à luz do cenário mundial evidencia a necessi dade de que esta dimensão química seja incorporada, no âmbito das perspectivas, na formulação da política de medicamentos no Brasil, que implica a necessidade de promover a abordagem interdisciplinar da política industrial, da política científica e tecnológica e da política de saúde, áreas nem sempre compatíveis entre si (Bermudez, 1995).

É necessário lembrar que, diferentemente da síntese química, que origina misturas racêmicas, os produtos obtidos por extração natural tendem a ser enantiomericamente puros. A riqueza de nossa biodiversidade também deve ser considerada, principalmente a médio e longo prazos e na elaboração de políticas setoriais de desenvolvimento industrial.

A propósito, cabe lembrar quando a Merck alemã, em 1971, instalou um laboratório em São Luiz do Maranhão, para produzir 30 toneladas/ ano (TA) de levodopa, por extração de uma planta da família das leguminosas conhecida como “mucuna-preta”. Esta planta contém apenas a forma levógira, o que permitiu à Merck competir com a Roche, cuja fábrica nos Estados Unidos, obtinha por síntese o produto racêmico. Como se trata de medicamento de uso contínuo para tratamento do Mal de Parkinson e causa sérios efeitos colaterais, não pode ser administrado na forma racêmica, razão por que a Roche tinha que separar o outro isômero, processo dispendioso. Desde 1986, outra empresa, a nacional "Produtos Vegetais do Piauí (PVP)", extrai da planta o fármaco, podendo produzir 24 TA em suas instalações.

Finalmente, é imperativo avaliar a capacidade instalada nas instituições universitárias e de pesquisa, que podem vir a se constituir em uma massa crítica necessária para impulsionar nosso desenvolvimento e subsidiar a formulação de diretrizes da política de Ciência \& Tecnologia nesta área do conhecimento. Caso contrário, estaremos abdicando de implementar uma política inserida em nossa realidade biosócio-cultural, aprofundando cada vez mais o processo de dependência.

\section{Referências}

BERMUDEZ, J. A. Z., 1992. Remédios: Saúdeou Indústria? A Produção de Medicamentos no Brasil. Rio de Janeiro: Relume Dumará.

BERMUDEZ, J. A. Z., 1994. Medicamentos genéricos: uma alternativa para o mercado brasileiro. Cadernos de Saúde Pública, 10:368-378.

BERM UDEZ, J. A. Z., 1995. Indústria Farmacêutica, Estado e Sociedade. Crítica da Política de Medicamentos no Brasil. São Paulo: HUCITEC/SOBRAVIME.

CHAN, A. S. C., 1993. A new route to important chiral drugs. CHEMTECH, 23:46-51.

DEUTSCH, D. H., 1991. Chiral drugs: The coming revolution. CHEMTECH, 20:157-159.

ELIEL, E. L., 1974. Perspectives in stereochemistry. CHEMTECH, 14:758-764.

FEDERSEL, H. J., 1993. Drug chirality - scale-up, manufacturing, and control. CHEMTEC, 23:24-33.

FERREIRA, V. F.; BARREIRO, E. J. L. \& COSTA, P. R. R., 1996. Substâncias enantiomericamente puras: Motivações acadêmicas e implicações industriais. Ciência Hoje (submetido a publicação).
STINSON, S. C., 1992. Chiral Drugs. Chemical \& Engineering News, 70:46-79.

STINSON, S.C., 1993. Chiral Drugs. Chemical \& Engineering News, 71:38-65.

STINSON, S.C., 1994a. Chiral Drugs. Chemical \& Engineering News, 72:38-72.

STINSON, S.C., 1994b. Market Environmental Pressures Spur Changes in Fine Chemicals Industry. Chemical \& Engineering News, 72:10-25.

WHO (World Health Organization), 1993a. Interchangeable multi-source pharmaceutical products. WHO draft guideline on marketing authorization requirements. Geneva: WHO. (Mimeo).

WHO (World Health Organization), 1993b. Fortysixth World Health Assembly - International nonproprietary names for pharmaceutical preparations. Report by the Director-General. Geneva: WHO. (Mimeo). 